\title{
Estado da arte da produção stricto sensu da enfermagem brasileira sobre saúde da população rural
}

\author{
State of the art of the brazilian nursing stricto sensu production on rural population health \\ Estado del arte de la producción stricto sensu de la enfermería brasileña sobre la salud de la población \\ rural
}

\author{
Bruno Neves da Silva ${ }^{1}$ (D) \\ Vitória Keller Gregório de Araújo² \\ Rayane Saraiva Felix ${ }^{1}$ (a)
}

Danielle Gonçalves da Cruz Rebouças ${ }^{2}$ (1)

Sandy Yasmine Bezerra e Silva ${ }^{1}(1)$

Erika Simone Galvão Pinto ${ }^{1}$ (1D

1. Universidade Federal do Rio Grande do

Norte, Programa de Pós-graduação em

Enfermagem. Natal, RN, Brasil.

2. Universidade Federal do Rio Grande do Norte. Natal, RN, Brasil.
Autor correspondente:

Bruno Neves da Silva.

E-mail: enfbneves@gmail.com.

Recebido em 18/11/2020.

Aprovado em 12/02/2021.

DOI:https://doi.org/10.1590/2177-9465-EAN-2020-0487

\begin{abstract}
Resumo
Objetivo: caracterizar a produção Stricto sensu da pós-graduação brasileira em Enfermagem sobre a temática saúde da população rural. Método: revisão compreensiva realizada por meio da Plataforma Sucupira. Estabeleceram-se, como amostra, dissertações e teses de mestrado e doutorado das quais foram extraídas informações que constituíram dois bancos de dados submetidos à análise estatística descritiva e textual. Resultados: 118 relatórios de pesquisa compuseram a amostra. Identificou-se maior quantitativo de estudos produzidos do tipo dissertação de mestrado, provenientes da região Sul do Brasil, publicados entre 2015 e 2016 e que utilizaram abordagens quantitativas. A análise exploratória permitiu constatar que as pesquisas desenvolvidas versavam sobre três temáticas centrais: políticas públicas e serviços de atenção à saúde, cuidado cultural no contexto familiar e saúde do idoso rural. Conclusões e implicações para a prática: a produção sobre a saúde rural em nível de pós-graduação Stricto sensu em Enfermagem no Brasil aponta para um novo leque de possibilidades de investigação e poderá contribuir para as práticas de cuidado que os enfermeiros rurais realizam.
\end{abstract}

Palavras-chave: Saúde da População Rural; Enfermagem; População Rural; Zona Rural; Educação de Pós-Graduação em Enfermagem.

\section{Abstract}

Objective: to characterize the Stricto sensu production of Brazilian post-graduate Nursing courses on the theme of rura population health. Method: comprehensive review carried out through the Sucupira Platform. The sample consisted of masters and doctoral dissertations and theses from which information was extracted to form two databases subjected to descriptive and textual statistical analysis. Results: A total of 118 research reports comprised the sample. We identified a higher number of studies produced in the form of master's dissertations, from the South region of Brazil, published between 2015 and 2016 , and that used quantitative approaches. The exploratory analysis found that the research developed focused on three central themes: public policies and health care services, cultural care in the family context, and health of the rural elderly. Conclusions and implications for practice: the production on rural health at the Stricto sensu post-graduate level in Nursing in Brazil points to a new range of possibilities for research and may contribute to the care practices that rural nurses perform.

Keywords: Rural Health; Nursing; Rural Population; Rural Areas; Education, Nursing, Graduate.

\section{RESUMEN}

Objetivo: caracterizar la producción Stricto sensu de los cursos brasileños de posgrado en enfermería sobre la salud de la población rural. Método: revisión comprensiva realizada a través de la Plataforma Sucupira. Se establecieron como muestra las disertaciones y tesis de maestría y doctorado, de las cuales se extrajo informaciones que constituyeron dos bases de datos sometidas al análisis estadístico descriptivo y textual. Resultados: 118 informes de investigación conformaron la muestra. Se identificó un mayor número de estudios producidos en el tipo de tesis de maestría, del Sur de Btrasil, publicados entre 2015 y 2016 y que utilizaron enfoques cuantitativos. El análisis exploratorio mostró que la investigación desarrollada abordó tres temas centrales: políticas públicas y servicios de salud, cuidado cultural en el contexto familiar y salud del anciano rural. Conclusiones e implicaciones para la práctica: la producción sobre salud rural a nivel de posgrado Stricto sensu en Enfermería en Brasi apunta a un nuevo abanico de posibilidades de investigación y puede contribuir a las prácticas de cuidado que realizan los enfermeros rurales.

Palabras clave: Salud rural; Enfermería; Población Rural; Medio Rural; Educación de Postgrado en Enfermería. 


\section{INTRODUÇÃO}

As populações rurais encontram-se inseridas no escopo das populações do campo, definidas pela Política Nacional de Saúde Integral das Populações do Campo e da Floresta (PNSIPCF) como aquelas que possuem seus modos de vida, produção e reprodução social relacionados majoritariamente com o campo, a floresta, os ambientes aquáticos, o extrativismo e a agropecuária. ${ }^{1}$

As populações que (re) existem na zona rural enfrentam iniquidades tanto relacionadas à difícil acessibilidade aos serviços e à baixa disponibilidade de profissionais de saúde para atender às suas demandas quanto a padrões de adoecimento diferenciados, com a persistência de padrões endêmicos e índices de qualidade de vida mais pobres que os da população em geral. ${ }^{2}$

A atuação da Enfermagem no meio rural é marcada por especificidades, pois envolve o desenvolvimento de um processo de trabalho que os profissionais geralmente não experienciaram durante a formação acadêmica, que é generalista, não contempla a realidade rural e não contribui para o rompimento do ciclo de invisibilização dessa população. ${ }^{3,4}$

Nessa perspectiva, a pesquisa em saúde rural emerge como um alicerce capaz de auxiliar a compreender a saúde rural e tem gradativamente ganhado espaço e solidez em um caminho que se apresenta como um instrumento de justiça social. É concebida por meio de métodos de diálogo de saberes que objetivam promover o empoderamento e a emancipação dos indivíduos. ${ }^{5}$ No entanto, apesar dos avanços que tem galgado, o desenvolvimento de estudos sobre a saúde da população rural ainda é pouco expressivo no Brasil, sobretudo, na área da Enfermagem. ${ }^{6-8}$

O reconhecimento da saúde rural, contudo, é premente para intervir no processo de saúde-doença no contexto da Atenção Primária à Saúde (APS), modalidade assistencial mais intimamente relacionada com o cuidado de saúde rural, e do Sistema Único de Saúde (SUS), pois o universo rural possui particularidades que condicionam a organização dos serviços de saúde e as práticas sanitárias e sociais. ${ }^{5}$ Para desenvolver políticas públicas adequadas e alcançar intervenções de saúde efetivas, necessita-se de conhecer as populações rurais, considerando as suas particularidades socioculturais e ambientais. ${ }^{9}$

Sob essa ótica, averiguar o conhecimento produzido no âmbito da pós-graduação, cenário intimamente relacionado à produção de estudos que contribuem diretamente para a elaboração de intervenções e políticas públicas que podem culminar com a melhoria da saúde e da qualidade de vida da coletividade, colabora para apreender essas particularidades que podem subsidiar a construção dessas políticas públicas voltadas à população rural.

Dessa forma, destaca-se a relevância deste estudo, que objetiva caracterizar a produção Stricto sensu da pós-graduação brasileira em Enfermagem sobre a saúde da população rural com vistas a delimitar o seu estado da arte. O estudo possibilita, ainda, contribuir para o avanço do conhecimento da Enfermagem enquanto ciência, profissão e disciplina acadêmica.

\section{MÉTODO}

Trata-se de um estudo de revisão compreensiva, de caráter documental e sistematizado. Sua operacionalização se deu a partir da seleção de descritores, buscas na literatura, seleção de relatórios de pesquisa para constituição da amostra, constituição de dois bancos de informações a partir da amostra e análise estatística descritiva e textual destes.

Os descritores, elencados a partir de consultas ao Descritores em Ciências da Saúde (DeCS), foram: "população rural", "saúde da população rural" e "zona rural". As buscas na base de dados escolhida foram realizadas entre janeiro e março de 2020 por dois pesquisadores independentes. Utilizou-se a Plataforma Sucupira e foi consultado o Catálogo de Dissertações e Teses da Coordenação de Aperfeiçoamento de Pessoal de Nível Superior (CAPES), o qual apresenta o panorama dos relatórios de pesquisa (dissertações e teses) dos programas de pós-graduação stricto sensu no Brasil. Nas buscas, utilizaram-se os descritores de forma isolada, sem associações.

Após a inserção dos descritores, foram aplicados os seguintes filtros: Grau acadêmico: mestrado, doutorado, mestrado profissional e doutorado profissional; e Área de conhecimento: Enfermagem. Para a seleção dos estudos que compuseram a amostra, foram incluídos os relatórios de pesquisa que se apresentaram disponíveis para o acesso na íntegra, ou aqueles que, apesar do ano da defesa ter ocorrido antes da criação da Plataforma Sucupira, encontraram-se disponíveis integralmente para livre acesso nos repositórios online das Universidades.

Como critério de exclusão, teve-se os relatórios de pesquisa que tratavam da saúde indígena, excluídos pelo entendimento de que, apesar de alguns povos habitarem em territórios rurais, possuem especificidades diferenciadas que constitui um campo de pesquisa específico na própria área de enfermagem. Não foi estabelecido recorte temporal na realização das buscas, dada a natureza do objetivo do presente estudo.

Após as buscas iniciais, selecionaram-se os estudos elegíveis para a amostra com base na leitura minuciosa dos títulos, seguida da leitura dos resumos e do texto na íntegra dos relatórios de pesquisa. Essas fases permitiram incluir ou excluir os estudos com base nos critérios de seleção elencados. Finalizada a seleção, realizou-se a criação de dois bancos de dados, para processamento das informações quantitativas e qualitativas presentes nos relatórios de pesquisa.

O primeiro banco de dados foi constituído de informações quantitativas extraídas das dissertações e teses, que representaram as variáveis Estado, Região e Instituição de Ensino Superior (IES) onde o relatório de pesquisa foi produzido, ano de defesa, nível acadêmico, abordagem, método para a coleta de dados e referencial teórico utilizados pelos autores. As informações foram digitadas em planilhas no software Microsoft Excel $2016 \AA$ e submetidas à análise estatística descritiva, com cálculo de 
frequências absolutas e relativas e posterior apresentação por meio de gráficos e tabelas.

O segundo banco de dados foi construído mediante a extração das seções de resultados e de conclusão dos resumos dos relatórios de pesquisa que compuseram a amostra. Utilizou-se a ferramenta Writer do software LibreOfficePortable $®$, que deu origem a um corpus textual, que foi posteriormente exportado para o processamento pelo software Interface de $R$ pour les Analyses Multidimensionnelles de Textes et de Questionnaires - IRAMUTEQ ${ }^{\circledR}$, para a análise estatística textual.

Utilizou-se o IRAMUTEQ $®$ para realizar a análise de classificação hierárquica descendente (CHD) e análise fatorial por correspondência (AFC). A CHD conduz análises quantitativas dos dados textuais e confecciona classes de conteúdos contextuais conforme a semelhança dos vocabulários. Por sua vez, a AFC permite visualizar, por meio de um plano cartesiano, como as palavras que compõem as classes se encontram próximas ou fazem oposição entre si, o que colabora para a delimitação de eixos temáticos de análise. ${ }^{10}$

Dessa forma, integradas as etapas de CHD e da AFC, a análise do corpus foi apresentada a partir de eixos temáticos abrangentes que permitiram delimitar o campo de pesquisa em saúde da população rural na pós-graduação stricto sensu em Enfermagem do Brasil e evidenciar as principais temáticas pesquisadas. $\mathrm{Na}$ apresentação de cada eixo, foram exibidos trechos extraídos do corpus, para fins de ilustração do conteúdo semântico que levaram às suas origens.

Ressalta-se que este estudo, devido utilizar exclusivamente textos científicos para revisão da literatura, dispensa apreciação ética, conforme os pressupostos da Resolução 510/2016 do Conselho Nacional de Saúde do Brasil. Nas ocasiões em que foram utilizados segmentos de texto provenientes dos relatórios consultados, estes foram identificados com a letra D (dissertação) ou T (tese), seguida por um número arábico aleatório.

\section{RESULTADOS}

Inicialmente, a estratégia de busca utilizada resultou em 22.878 relatórios de pesquisa. Após o refinamento inicial baseado na leitura dos títulos, foram pré-selecionadas 304 , submetidos à leitura dos resumos, restando, após isso, 137 relatórios de pesquisa, submetidos à análise na íntegra, o que permitiu estabelecer 118 dissertações e teses como amostra. O restante dos estudos foi excluído por não se enquadrar nos critérios de seleção. A Figura 1 esquematiza esse processo.

Quanto ao intervalo temporal, $44,1 \%$ ( $n=52$ ) dos estudos foram publicados no intervalo de anos entre 2012 a 2015, conforme pode-se constatar na série histórica representada na Figura 2.

Cerca de 47,5\% ( $n=55)$ dos estudos foram desenvolvidos em programas de pós-graduação localizados na região Sul do país, seguida pela região Sudeste, com $33,1 \%$ ( $n=38)$. A universidade com maior número de pesquisas desenvolvidas foi a Universidade Federal de Minas Gerais. A Figura 3 evidencia o quantitativo de estudos desenvolvidos por região do Brasil e universidade.

No tocante ao nível acadêmico, houve a predominância de dissertações de mestrado, que representaram $68,6 \%(n=82)$ da amostra. Quanto aos aspectos metodológicos, as abordagens utilizadas equipararam-se, com leve predileção pela abordagem quantitativa, correspondendo a 50,8\% ( $n=57)$. Cerca de $95 \%$ $(n=112)$ realizaram entrevista como uma das formas de coleta de dados (ressalta-se a utilização de mais de um método de coleta de dados na maioria dos estudos). Em relação ao referencial teórico utilizado, apesar de $63,5 \%$ ( $n=75)$ não o terem especificado, destaca-se a utilização da teoria transcultural por $7,6 \%(n=9)$, conforme se observa na Tabela 1.

No tocante ao processamento do corpus textual, constatou-se a ocorrência de 15.454 palavras, com 3.253 formas distintas e 430 segmentos de texto (STs - partições que o software realiza

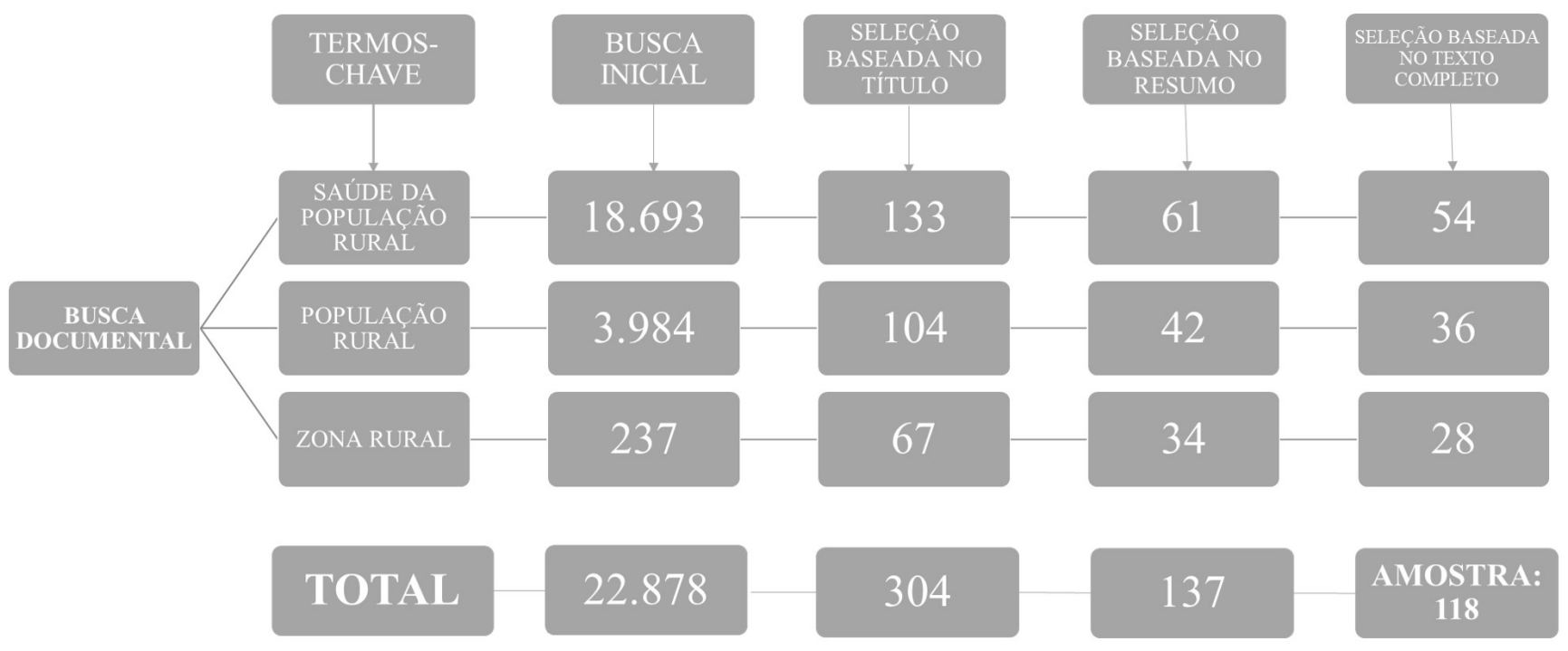

Figura 1. Passos integralizados para a constituição da amostra do estudo. Natal (RN), 2020. Fonte: dados da pesquisa, 2020. 


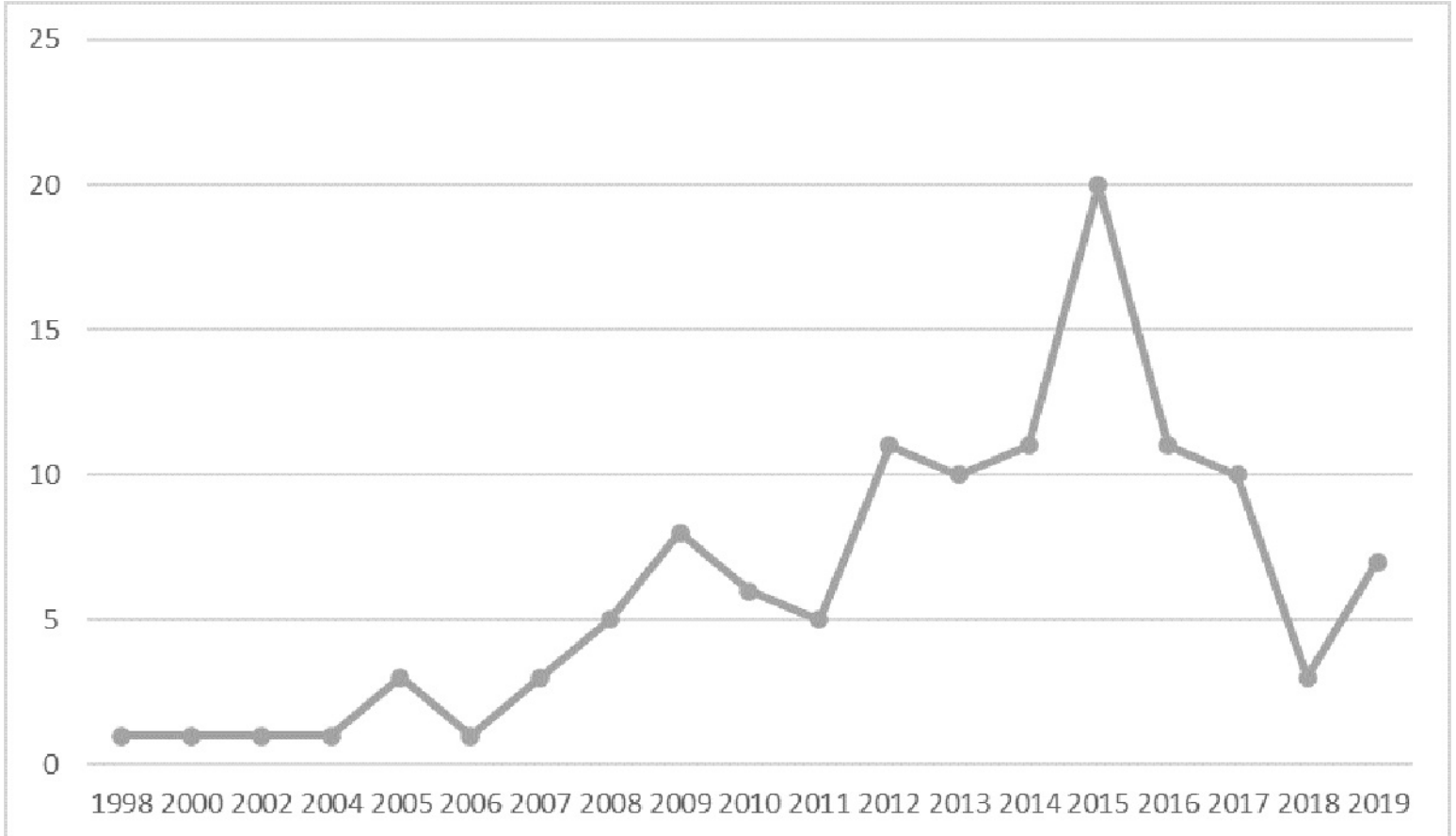

Figura 2. Intervalo temporal de publicação das dissertações e teses em Enfermagem sobre a saúde da população rural que compuseram a amostra. Natal, RN, 2020.

Fonte: dados da pesquisa, 2020.

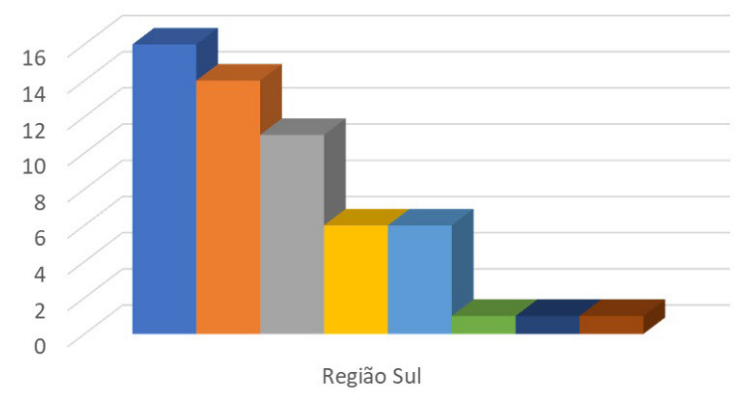

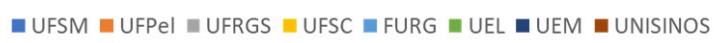
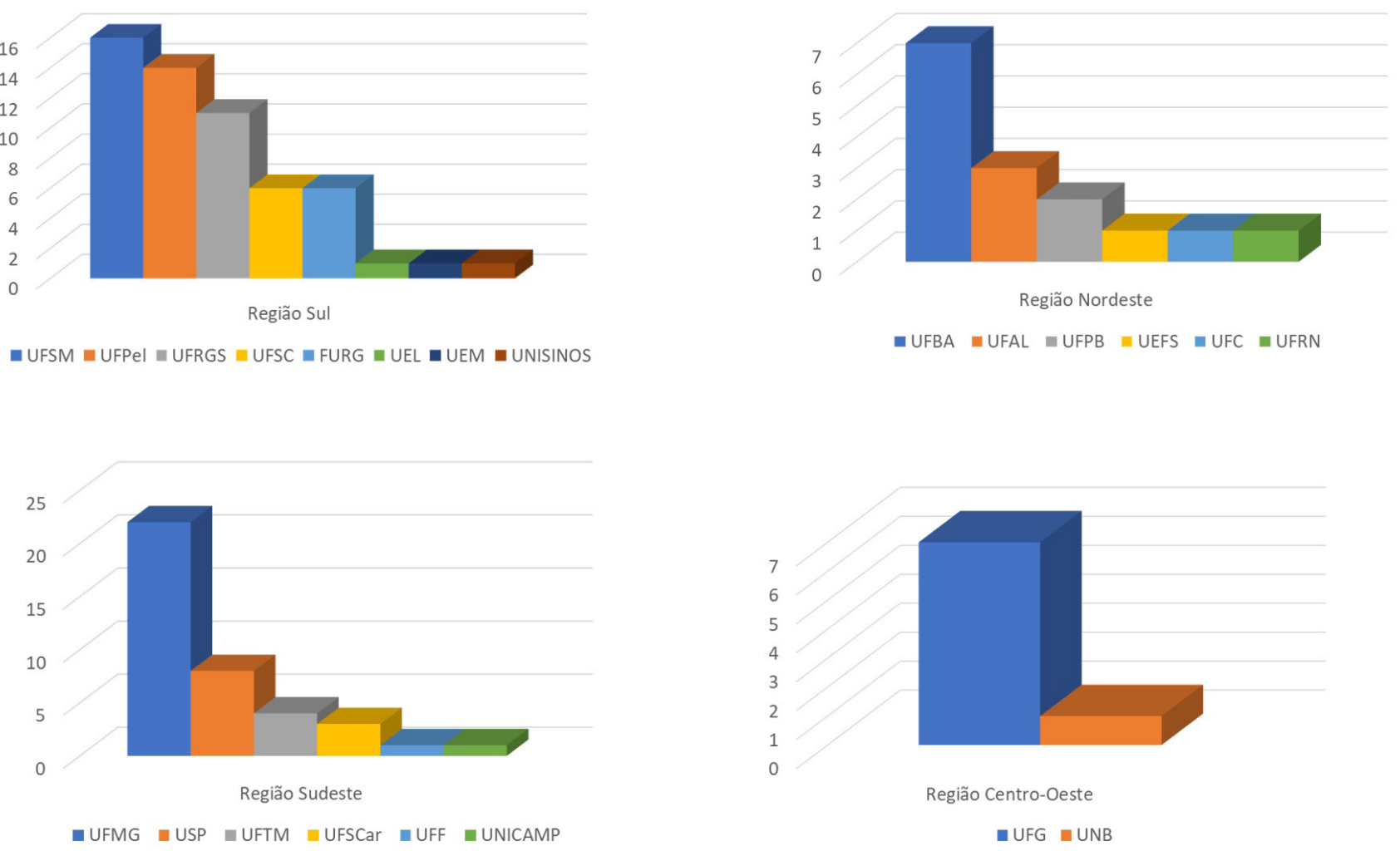

Região Centro-Oeste

- UFG $\square \mathrm{UNB}$

Figura 3. Produção de dissertações e teses em saúde da população rural segundo universidade e região do Brasil. Natal, RN, 2020.

Fonte: dados da pesquisa, 2020. 
no corpus para efetuar as análises, geralmente com três linhas de tamanho), com média de 4,75 ocorrências para cada forma de palavra (valor médio utilizado para definir as palavras a serem incluídas nas classes do dendrograma construído pelo método da CHD). Cerca de 1.844 palavras apareceram apenas uma única vez (hápax). A análise lexicográfica do corpus obteve a retenção de 89,77\% (o que corresponde a 386 de 430 STs), considerada satisfatória para fins de análise.

A CHD considerou, para a construção das classes, os seguintes critérios: palavras cuja frequência foi maior que 0 dobro da média de ocorrências no corpus $(F>9,5)$; palavras com valor de qui-quadrado $\left(\chi^{2}\right)$ maior ou igual a 3,84 (significância de $95 \%$ e e que apresentaram significância estatística ( $p$-valor $\leq 0,05$ ).

Conforme pode ser observado na Figura 4, durante a CHD, o corpus foi inicialmente subdividido em dois subcorpus, um deles dando origem à Classe 5 , composta por 55 STs. Posteriormente, o corpus da direita foi dividido em dois, constituindo as Classes 3 , com 88 STs, e 4, com 86 STs. Em uma última etapa, em oposição às Classes 3 e 4, ocorreu uma última partição no corpus, que deu origem às Classes 1 , com 98 STs, e 2, com 59 STs.

Analisou-se cada conjunto semântico de palavras que compôs cada uma das classes lexicais apontadas pela CHD e, com o apoio da AFC, percebeu-se uma semelhança de conteúdo e a íntima relação entre as Classes 1 e 2 e entre as Classes 3 e 4. Constatou-se, ainda, que as palavras que constituíram a Classe 5 encontraram-se mais afastadas das demais classes, enquanto as palavras das Classes 1 e 2 e as das Classes 3 e 4 associaramse intimamente.

A análise do dendrograma proveniente da CHD, juntamente com as oposições e as semelhanças visualizadas na AFC, permitiu constatar que a junção das Classes 1 e 2 possibilitou formular o primeiro eixo temático, intitulado Políticas públicas e serviços de atenção à saúde rural. As Classes 3 e 4, por sua vez, permitiram elaborar um segundo eixo temático denominado Cuidado cultural no contexto da família rural. Por fim, a Classe 5 , separadamente, deu origem a um terceiro eixo designado Saúde do idoso rural.

Tais eixos possibilitaram delimitar o estado da arte das pesquisas sobre a saúde rural realizadas pela Enfermagem no Brasil, identificando, de maneira generalista, o panorama temático da produção Stricto sensu sobre essa área do conhecimento.

O primeiro eixo temático examina as políticas públicas voltadas para a população rural desenvolvidas no Brasil, salientando o seu desenvolvimento tardio e incipiente, e a invisibilização dessa população diante do poder público; discorre, também, sobre as barreiras enfrentadas pelas comunidades rurais no acesso e a acessibilidade aos serviços de saúde e sobre o papel da APS no cuidado à saúde rural. Os trechos a seguir ilustram essas análises.

\section{[...] no Brasil, vêm sendo criadas políticas para a redução} das desigualdades em saúde dos diversos grupos de populações_rurais do país, mas essas políticas não se efetivaram completamente, e a população_rural tem condições de vida e saúde precárias [...] (T01).
Tabela 1. Caracterização das variáveis selecionadas dos relatórios de pesquisa que constituíram a amostra. Natal, RN, 2020. $(n=118)$.

\begin{tabular}{|c|c|c|}
\hline Variável & $\mathbf{N}$ & $\%$ \\
\hline \multicolumn{3}{|l|}{ Nível acadêmico } \\
\hline Mestrado & 81 & 68,6 \\
\hline Doutorado & 36 & 30,5 \\
\hline Mestrado profissional & 1 & 0,8 \\
\hline \multicolumn{3}{|l|}{ Abordagem } \\
\hline Quantitativa & 60 & 50,8 \\
\hline Qualitativa & 50 & 42,3 \\
\hline Mista & 8 & 6,8 \\
\hline \multicolumn{3}{|l|}{ Método de coleta de dados } \\
\hline Entrevista & 115 & 97,5 \\
\hline Observação & 26 & 22,0 \\
\hline Exame clínico & 15 & 12,7 \\
\hline Exame laboratorial & 13 & 11,0 \\
\hline Documental & 7 & 5,9 \\
\hline Genograma & 6 & 5,1 \\
\hline Grupo focal & 4 & 3,4 \\
\hline Diário de campo & 4 & 3,4 \\
\hline Fotografia & 4 & 3,4 \\
\hline História oral & 3 & 2,5 \\
\hline Inquérito domiciliar & 2 & 1,7 \\
\hline Ecomapa & 2 & 1,7 \\
\hline Evocação livre de palavras & 2 & 1,7 \\
\hline Círculos de cultura & 1 & 0,8 \\
\hline Mapa corporal & 1 & 0,8 \\
\hline Gibi & 1 & 0,8 \\
\hline Levantamento etnobotânico & 1 & 0,8 \\
\hline Photovoice & 1 & 0,8 \\
\hline Técnica do incidente crítico & 1 & 0,8 \\
\hline Rede de relações & 1 & 0,8 \\
\hline \multicolumn{3}{|l|}{ Referencial teórico utilizado } \\
\hline Não especificado & 77 & 65,3 \\
\hline Teoria transcultural & 9 & 7,6 \\
\hline Etnoenfermagem & 6 & 5,1 \\
\hline Interacionismo simbólico & 4 & 3,4 \\
\hline Educação crítica libertadora & 3 & 2,5 \\
\hline Determinantes Sociais de Saúde & 2 & 1,7 \\
\hline Antropologia interpretativa & 2 & 1,7 \\
\hline Teoria das Representações Sociais & 2 & 1,7 \\
\hline Teoria bioecológica & 2 & 1,7 \\
\hline Teoria do desenvolvimento da família & 2 & 1,7 \\
\hline Teoria geral dos sistemas & 2 & 1,7 \\
\hline Filosofia do cuidado de Enfermagem & 1 & 0,8 \\
\hline Teorias de gênero & 1 & 0,8 \\
\hline Racionalidade cultural & 1 & 0,8 \\
\hline Educação popular em saúde & 1 & 0,8 \\
\hline Sociedade de risco & 1 & 0,8 \\
\hline Espaço social alimentar & 1 & 0,8 \\
\hline Fenomenologia & 1 & 0,8 \\
\hline TOTAL & 118 & 100 \\
\hline
\end{tabular}

Fonte: Dados da pesquisa, 2020 


\begin{tabular}{|c|c|c|c|c|c|c|c|c|c|c|c|c|c|c|}
\hline & & & & \multicolumn{6}{|c|}{ TRATAMENTO DO CORPUS - 89,77\% (386 STs) } & & & & & \\
\hline \multicolumn{3}{|c|}{ CLASSE $5-55$ STs $-14,2 \%$} & \multicolumn{3}{|c|}{ CLASSE $2-59$ STs $-15,3 \%$} & \multicolumn{3}{|c|}{ CLASSE $1-98$ STs $-35,39 \%$} & \multicolumn{3}{|c|}{ CLASSE $4-86$ STs - 22,28\% } & \multicolumn{3}{|c|}{ CLASSE $3-88$ STs - 22,8\% } \\
\hline Idoso & 40 & 46,68 & Saúde & 151 & 10,02 & Rural & 112 & 36,87 & Trabalho & 45 & 7,07 & Cuidado & 69 & 139,27 \\
\hline Apresentar & 24 & 26,77 & Serviço & 53 & 73,77 & Ação & 36 & 19,07 & Familiar & 43 & 10,71 & Familia & 53 & 35,56 \\
\hline Alto & 16 & 61,33 & Profissional & 36 & 7,15 & Necessidade & 31 & 6,96 & Doença & 38 & 35,61 & Familiar & 43 & 22,12 \\
\hline Elevado & 16 & 35,1 & Necessidade & 31 & 10,62 & Público & 29 & 14,68 & Vida & 37 & 19,97 & Prático & 38 & 39,01 \\
\hline Tratamento & 16 & 7,39 & Acesso & 30 & 36,37 & Político & 25 & 30,66 & Relação & 36 & 14,26 & Meio & 30 & 10,53 \\
\hline Nivel & 14 & 57,14 & Utilizar & 23 & 43,48 & Área & 22 & 33,15 & Social & 35 & 9,41 & Comunidade & 29 & 8,65 \\
\hline \multirow[t]{4}{*}{ Depressão } & 11 & 54,46 & Ausência & 11 & 20,44 & Desenvolvimento & 12 & 7,1 & Cultural & 21 & 15,59 & Cotidiano & 16 & 7,02 \\
\hline & & & & & & Falta & 11 & 13,39 & Experiência & 18 & 16,41 & Principal & 15 & 8,27 \\
\hline & & & & & & Direcionar & 11 & 8,74 & Cotidiano & 16 & 7,41 & Cuidar & 13 & 36,94 \\
\hline & & & & & & & & & Compreensão & 10 & 8,44 & Diverso & 11 & 16,04 \\
\hline
\end{tabular}

Figura 4. Dendrograma proveniente da CHD efetuada pelo IRAMUTEQ no corpus textual do estudo. Natal, RN, 2020. Fonte: elaboração própria, 2020.

[...] a saúde das populações_rurais é um fenômeno amplo, com suas especificidades, e o desafio das políticas_públicas é o de reconhecer o cenário rural como espaço de cuidado que demanda intervenções singulares $[\ldots]$ (T02).

[...] o acesso é prejudicado em duas formas: a longa distância geográfica das microáreas a unidade_de_saúde, ligada à insuficiência de veículo para a equipe, e pela limitação de consultas médicas [...] (T03)

[...] as condições necessárias para o alcance de uma vida mais saudável para a população_rural envolve melhorias na Atenção_Primária_à_Saúde e os profissionais envolvidos [...] (D01).

O segundo eixo temático delimitou o cuidado cultural no contexto às famílias rurais desenvolvido pela Enfermagem, que engloba a capacidade do enfermeiro de incorporar a cultura dos sujeitos rurais no desenvolvimento da sua prática, alinhando saberes populares e científicos, o que é permeado por entraves que se relacionam à deficiência no processo formativo do enfermeiro para a atuação em âmbitos rurais, conforme pode ser visualizado nos trechos que seguem.

[...] a enfermagem necessita promover uma assistência que considere os valores e crenças dos sujeitos a partir da compreensão da doença não apenas como um processo biológico, mas social, cultural e político [...] (D02).
[...] o preparo formal recebido na graduação oportunizou um aprendizado genérico para trabalhar com as pessoas, mas não foi contextualizado o modo de vida destas populações (rurais) [...] (T04).

O terceiro eixo temático analisa a saúde do idoso rural e destaca a acessibilidade dos idosos rurais aos serviços de saúde, bem como pincela sobre a prevalência de agravos relevantes em idosos do meio rural. Os trechos a seguir ilustram essas inferências.

[...] os principais motivos para os idosos não recorrerem aos serviços_de_saúde mesmo em caso de necessidade estão relacionados aos recursos financeiros insuficientes, demora no atendimento, ausência de transporte, uso de automedicação e ausência de profissionais médicos [...] (D03)

[...] as condições de pré_fragilidade e fragilidade apresentaram elevado percentual de ocorrência associadas a maiores chances para desfechos adversos à saúde e repercussão negativa na qualidade_de_vida de idosos (D04).

[...] existe um número mais elevado de homens idosos do que de mulheres no meio rural e de forma geral uma inserção socioeconômica precária, baixa escolaridade, renda familiar reduzida e segregação em espaços rurais delimitados por latifúndios (D05). 


\section{DISCUSSÃO}

Na área da saúde, são poucos os trabalhos que avaliam as condições gerais ou mais de um indicador de saúde de populações rurais em cenários regionais ou nacionais, e os estudos existentes concentram-se em investigações de aspectos específicos da saúde do trabalhador rural. ${ }^{9}$ Tal fato é corroborado também no âmbito da Enfermagem. ${ }^{3}$

Este estudo evidenciou que o quantitativo de estudos desenvolvidos teve um avanço no ano de 2015, o que pode estar relacionado ao fato de, em 2013, ter sido publicada, pelo Ministério da Saúde, a PNSIPCF, ${ }^{1}$ levando-se em consideração que a maioria das pesquisas de mestrado, que foram as mais realizadas, levam cerca de dois anos até à sua conclusão.

Apesar do avanço obtido, o desenvolvimento de estudos sobre as populações rurais no âmbito da Enfermagem do Brasil ainda é pouco expressivo. A lacuna, contudo, desponta como uma nova possibilidade de investigação devido à necessidade de ampliação do conhecimento sobre a temática, que pode contribuir para avanços na atuação do enfermeiro rural e para o estabelecimento da sua identidade profissional no Brasil, ${ }^{6}$ posto que estudos sobre essa identidade ainda são escassos.

Para mais, a caracterização do conhecimento da Enfermagem sobre a saúde rural pode impulsionar o avanço da prática e da profissão, abrindo janelas, inclusive, para interfaces com a Prática Avançada de Enfermagem, um instrumento inovador que pode colaborar para a melhoria da atenção à saúde de grupos populacionais de maior vulnerabilidade ou de difícil acesso, como no caso da população rural. ${ }^{11}$

Quanto à caracterização dos estudos identificados, as abordagens utilizadas ressaltam a existência de domínios quantificáveis e não quantificáveis ou qualificáveis e que o fenômeno de interesse (nesse caso, a saúde da população rural) ora precisa ser decomposto, ora precisa ser recomposto em um todo e que, nesse processo, existem pretensões tanto mensurativas, como descritivas, compreensivas, analíticas, classificatórias, dentre outras, o que explica a quase equiparação entre as abordagens. ${ }^{12}$ A predileção pela abordagem quantitativa, no entanto, também pode ter sofrido influência da concepção mais pós-positivista dos pesquisadores. ${ }^{13}$

No que concerne aos procedimentos para a coleta de dados, sobressai-se a utilização da entrevista em quase a totalidade da amostra, explicada devido à sua operacionalização ser adequada para estudos tanto qualitativos quanto quantitativos, bem como devido à sua ligação mais formal com a fase de coleta de dados. ${ }^{12}$

Quanto aos referenciais teóricos utilizados nos estudos, apesar de a maioria não ter especificado, destaca-se a utilização da Teoria Transcultural, que representa um dos referenciais específicos da própria Enfermagem que mais vem sendo utilizado pelos pesquisadores. ${ }^{14}$

A delimitação temática evidenciou pouca variabilidade dos temas de estudos desenvolvidos pela Enfermagem sobre a sua própria atuação frente à saúde da população rural, estando os estudos mais relacionados a temas gerais na área da saúde pública. Tal fato já foi reiterado por outros autores. ${ }^{6}$

Em relação aos achados enquadrados no primeiro eixo temático, sabe-se que, no Brasil, as políticas de saúde evoluíram juntamente das tendências políticas e econômicas, seguindo, sobretudo, o desenvolvimento do capitalismo, o que colocou a perspectiva da saúde pública em segundo plano. As populações rurais, nesse contexto, eram invisíveis aos olhos das políticas de saúde. ${ }^{15}$

No entanto, é de conhecimento público que essa população requer, há muito tempo, a atenção dessas políticas, sobretudo do SUS, devido às iniquidades em saúde existentes e para colaborar com a superação do modelo de atenção vigente, que não considera as especificidades dos sujeitos rurais. ${ }^{16}$ Tais políticas, não necessariamente específicas do setor de saúde, estabelecem conexões com aspectos intrínsecos à vida dos sujeitos e implicam amplamente o processo saúde-doença. ${ }^{17}$

A evolução histórica das políticas públicas em meio rural demonstra que os direitos trabalhistas e de saúde dessa população foram tardiamente reconhecidos. Com a criação do Fundo de Assistência e Previdência ao Trabalhador Rural (FUNRURAL), em 1963 (posteriormente incorporado ao INAMPS em 1974), a partir da Lei do Estatuto do Trabalhador Rural, formalizou-se o oferecimento de assistência médica para o trabalhador rural mediante a contribuição previdenciária. $\mathrm{O}$ acesso universal à saúde por esses indivíduos, no entanto, deu-se somente com a criação do SUS e foi intensificado com investimentos na APS e nas equipes de Saúde da Família. ${ }^{18,19}$

Inexiste, no Brasil, uma política de APS voltada especificamente para o território rural, ainda que seja esse o modelo assistencial predominante que assiste os indivíduos contidos nele. Apesar da existência da PNSIPFC, na prática, essa política não se articula com a Política Nacional de Atenção Básica do país, aplicada genericamente para populações urbanas e rurais. ${ }^{20}$ Como fator agravante, tem-se o fato da incipiência de participação da população rural na formulação dessas políticas públicas. ${ }^{21}$

A ineficiência e a insuficiência dessas políticas reflete a situação dos serviços rurais de atenção à saúde no Brasil. A oferta de cuidados de saúde é baseada no trabalho das equipes de Saúde da Família que, apesar do potencial de reorganizar o modelo assistencial curativista, atuam com base na priorização da demanda espontânea, concedem pouca autonomização aos usuários e findam por transformar-se em locais burocráticos que atuam como "pontes" para o indivíduo acessar outros níveis de atenção. ${ }^{16,22,23}$

Outras características desses serviços são a dificuldade de acesso pelos usuários que não possuem transporte próprio, a insuficiência de insumos básicos, a precariedade da estrutura física dos estabelecimentos de atenção à saúde existentes e a baixa disponibilidade de profissionais. Existe, ainda, uma limitação de turnos de horário de atendimento, o que provoca vazios assistenciais e compromete a integralidade do cuidado desenvolvido. ${ }^{20,22,24-26}$

Ainda que, com o advento do SUS, importantes avanços tenham sido conquistados e, nos últimos anos, tenha ocorrido um reconhecimento da população rural perante as políticas públicas, a plenitude do alcance desses direitos não está garantida, posto que existem vazios assistenciais e grandes distâncias a serem percorridas na busca pela oferta e pelo acesso aos serviços de saúde. ${ }^{18}$ 
Nessa perspectiva, a organização do trabalho da Enfermagem na Estratégia Saúde da Família (ESF) deve ser organizada de forma a estimular a participação popular nos conselhos, debates e reuniões locais de forma a permitir que a população rural participe da elaboração, programação e fiscalização das políticas públicas e contribua para o aumento da eficácia e abrangência das ações de saúde. ${ }^{3}$

O segundo eixo temático examina o cuidado cultural no contexto da família rural. Os estudos desenvolvidos pela Enfermagem acerca dessa temática são abordados mediante perspectivas ecológicas e etnográficas, que possibilitam desvelar a construção de uma realidade sobre a saúde e a doença das famílias rurais, ${ }^{27}$ o que compreende o estabelecimento de relações entre o morrer e o adoecer com o estilo de vida e o tipo de trabalho, que são diretamente influenciados por fatores culturais. ${ }^{24}$

As famílias rurais cuidam-se por intermédio de crenças, valores e hábitos construídos a partir do processo de interação entre os seus próprios membros e entre toda a comunidade rural, que se cristalizaram e vão sendo transmitidos entre os sujeitos de acordo com o contexto pelo qual transitam de forma individual. ${ }^{28}$ Dessa forma, o cuidado presente na vida das famílias rurais é concebido como um cuidado cultural, pois a estrutura sociocultural e as visões de mundo apropriadas influenciam suas práticas de cuidado à saúde. ${ }^{29}$

Nessa perspectiva, sob a ótica da família rural, a saúde não significa apenas a ausência de dor, mas se relaciona às práticas cotidianas, e o cuidado à saúde encontra-se associado principalmente à alimentação e ao cuidado familiar, que majoritariamente é responsabilidade das mulheres que compõem essas famílias, e seus rituais de cuidado envolvem utilizar tanto fármacos prescritos quanto plantas medicinais e práticas voltadas para a religiosidade. ${ }^{30}$

Logo, para exercer o cuidado diante das famílias rurais, os enfermeiros precisam compreender o contexto cultural da comunidade que acompanham, entender e valorizar os saberes locais, juntamente com seus comportamentos, práticas e dinâmicas de cuidado que integram seu sistema de cuidado à saúde. É preciso ainda abranger a sua atuação em diferentes manifestações de saber além do conhecimento biologicista, de forma a confluir os conhecimentos empíricos dos membros das famílias com o saber científico, e buscar um ponto de equilíbrio que permita superar os entraves e construir um modelo rural de saúde eficaz. ${ }^{3,30-32}$

Para mais, os enfermeiros devem visualizar as famílias rurais com ênfase sobre as mudanças de seu ciclo vital e apostar na educação em saúde durante as visitas domiciliares e nos espaços da própria comunidade, promovendo uma aproximação com as famílias de forma a possibilitar um cuidado compartilhado que leve em consideração as potencialidades e fragilidades do cuidado familial. ${ }^{27}$

No entanto, ainda que o cuidado à família rural se constitua em um campo de atuação para a Enfermagem, principalmente no contexto da ESF, os enfermeiros que atuam na assistência à saúde rural enfrentam dificuldades relacionadas à formação acadêmica insuficiente para atuarem nesses cenários, tendo-se em vista que as escolas de Enfermagem enfocam suas aulas práticas e estágios em cenários urbanos e, dessa forma, os enfermeiros não conseguem captar, durante a sua formação, os modos de cuidar pautados na diversidade cultural dos sujeitos. ${ }^{3,27}$

Diante desses desafios, países como Austrália, Estados Unidos da América e Canadá têm impulsionado a organização da prática em Enfermagem rural a partir de mudanças em legislações e da ampliação da atuação dos enfermeiros com a integração entre educação, pesquisa, gestão e prática assistencial. ${ }^{4}$

Dessa forma, ressalta-se a importância de o Brasil seguir esses exemplos e de adotar medidas como a de ofertar, aos discentes dos cursos de Enfermagem, a oportunidade de experenciar, durante a sua formação, vivências na ESF rural, o que permite exercitar o pensamento reflexivo, a expressão simbólica, melhorar o processo de comunicação e desenvolver capacidades criativas, exercendo um papel transformador que contribuirá para a atuação no âmbito rural após a formação. ${ }^{4}$

Por fim, o terceiro eixo temático delimita a produção sobre a saúde do idoso rural pela Enfermagem. Os idosos rurais estão susceptíveis a condições clínicas agravantes diferentes das daqueles que residem em zona urbana e isso ocorre principalmente devido aos fatores ambientais aos quais estão expostos e devido ao acesso à saúde ser mais difícil e menos prioritário na percepção deles, já que a maioria precisa trabalhar no campo durante o dia para sobreviver. ${ }^{33}$

O fato de os idosos residirem na zona rural acentua naturalmente as vulnerabilidades pelas quais perpassam e estudos demonstram que existem características comuns entre países em desenvolvimento e países desenvolvidos, como a menor média de rendimento e a maior severidade nos problemas de saúde. ${ }^{34}$

Pesquisadores discorrem que o uso regular de serviços de saúde foi maior entre os idosos do meio urbano quando comparados aos idosos do meio rural, indicando a situação de desvantagem no acesso e na utilização dos serviços de saúde pela população rural, por exemplo. ${ }^{35}$

Estima-se que a oferta de serviços de saúde para idosos residentes no meio rural seja difícil por características relativas ao acesso (transporte insuficiente, condições ruins das estradas ou faltas delas, localidade distante dos serviços de saúde, isolamento social), à renda e ao próprio hábito dos idosos em procurar assistência curativa/terapêutica e não preventiva. ${ }^{33,36}$

As barreiras geográficas da zona rural, sobretudo, constituemse em um fator impeditivo para o maior acesso aos serviços de saúde dessa população. Esse fator dificulta o acompanhamento adequado dos problemas de saúde existentes, propiciando a ocorrência de complicações, que, por sua vez, têm impacto negativo na qualidade de vida do idoso, ${ }^{37}$ que, apesar dos entraves impostos, tem apresentado índices mais elevados do que entre os idosos residentes em zona urbana, ${ }^{38}$ porém, encontra-se influenciada pela presença de morbidades e/ou incapacidade funcional. ${ }^{39,40}$

Destacam-se, entre as morbidades que afetam os idosos rurais, a depressão, cuja prevalência de $8,1 \%$ já foi detectada por um estudo de base populacional, o Diabetes Mellitus, cuja prevalência de $16,9 \%$ já foi evidenciada no Estado do Rio Grande do Sul, por exemplo, e a síndrome da fragilidade do idoso rural, que possui prevalência constatada na literatura de cerca de $43 \%$. Dentre os fatores que contribuíram para esses agravos, teve-se a baixa escolaridade em comum. ${ }^{41-43}$ 
Salienta-se que o grupo etário idoso é aquele com menor índice de escolaridade no Brasil, o que é acentuado em regiões rurais, e aponta para a necessidade de políticas educacionais que culminem com o empoderamento dessa população, tanto para o cuidado de si quanto para a promoção da sua saúde. ${ }^{44}$

Nessa perspectiva, destaca-se que as demandas do processo de envelhecimento no meio rural brasileiro são caracterizadas por um conjunto de demandas específicas, as quais requerem uma atenção especial por parte de diferentes setores, sobretudo os da saúde. ${ }^{35}$

Necessita-se de estabelecer, portanto, um planejamento de saúde aos idosos que habitam nessas áreas. Nesse sentido, a introdução e a implementação do cuidado a essa população devem ser garantidas a partir da integralidade da assistência, da promoção da saúde e da prevenção de agravos, assim como mencionado nas diversas políticas de saúde que norteiam o SUS. ${ }^{45}$

Reforça-se, dessa forma, a importância do papel do enfermeiro na atuação do cuidado à pessoa idosa, o que corrobora a elucidação do perfil e o estilo de vida dos idosos rurais, com vistas a intervir nos problemas associados à fragilidade e atuar na prevenção e postergação de desfechos adversos e indesejáveis. ${ }^{43,46}$

\section{CONCLUSÕES E IMPLICAÇÕES PARA A PRÁTICA}

A análise dos relatórios de pesquisa permitiu desvelar que a saúde da população rural ainda é um campo de estudos pouco explorado em nível de pós-graduação Stricto sensu em Enfermagem no Brasil. O estado da arte, nesse campo de pesquisa, demonstrou pouca variabilidade das temáticas, que se concentraram, genericamente, em estudos sobre políticas públicas e serviços de atenção, cuidado cultural no contexto familiar e saúde do idoso rural.

No entanto, ressalta-se a importância do desenvolvimento, pela Enfermagem, de estudos que abordem a saúde da população rural, tanto devido à lacuna existente sobre a sua atuação nesses contextos quanto devido às suas particularidades $\mathrm{e}$ vulnerabilidades requererem atenção, o que aponta para um leque de possibilidades de investigação para os pesquisadores devido à necessidade de ampliação do conhecimento nesse campo de estudos.

Destaca-se, ainda, a necessidade desses estudos para a prática profissional da Enfermagem em âmbito rural, pois explorar os estudos que a Enfermagem desenvolve sobre essa temática, bem como as práticas de cuidado que os enfermeiros rurais realizam, contribui para nortear as ações dos enfermeiros, bem como para aumentar o corpo de conhecimentos da ciência da Enfermagem.

Quanto às limitações deste estudo, destaca-se a não possibilidade de recuperação de alguns relatórios de pesquisas via Plataforma Sucupira ou pelos Repositórios Institucionais.

\section{CONTRIBUIÇÕES DOS AUTORES}

Desenho do estudo. Bruno Neves da Silva. Erika Simone Galvão Pinto.
Coleta ou produção dos dados. Bruno Neves da Silva. Vitória Keller Gregório de Araújo. Erika Simone Galvão Pinto.

Análise de dados. Bruno Neves da Silva. Vitória Keller Gregório de Araújo. Rayane Saraiva Felix. Erika Simone Galvão Pinto.

Interpretação dos resultados. Bruno Neves da Silva. Vitória Keller Gregório de Araújo. Rayane Saraiva Felix. Danielle Gonçalves da Cruz Rebouças. Sandy Yasmine Bezerra e Silva. Erika Simone Galvão Pinto.

Redação e revisão crítica do manuscrito. Bruno Neves da Silva. Vitória Keller Gregório de Araújo. Rayane Saraiva Felix. Danielle Gonçalves da Cruz Rebouças. Sandy Yasmine Bezerra e Silva. Erika Simone Galvão Pinto.

Aprovação da versão final do artigo. Bruno Neves da Silva. Vitória Keller Gregório de Araújo. Rayane Saraiva Felix. Danielle Gonçalves da Cruz Rebouças. Sandy Yasmine Bezerra e Silva. Erika Simone Galvão Pinto.

Responsabilidade por todos os aspectos do conteúdo e a integridade do artigo publicado. Bruno Neves da Silva. Vitória Keller Gregório de Araújo. Rayane Saraiva Felix. Danielle Gonçalves da Cruz Rebouças. Sandy Yasmine Bezerra e Silva. Erika Simone Galvão Pinto.

\section{EDITOR ASSOCIADO}

Rodrigo Nogueira da Silva

\section{REFERÊNCIAS}

1. Ministério da Saúde (BR). Política Nacional de Saúde Integral das Populações do Campo e da Floresta [Internet]. Brasília, DF: Editora do Ministério da Saúde; 2013 [citado 2020 nov 18]. Disponível em: https://bvsms.saude.gov.br/bvs/publicacoes/politica_nacional_saude_ populacoes_campo.pdf

2. Castilho EA, Gonçalves H. Problemas de saúde e a zona rural. Rev Saude Publica. 2018;52(Supl. 1):1s-2s. http://dx.doi.org/10.11606/ S1518-8787.20180520supl1ed.

3. Silva EM, Portela RA, Medeiros ALF, Cavalcante MCW, Costa RTA. Os desafios no trabalho da enfermagem na Estratégia Saúde da Família em área rural: revisão integrativa. Hygeia. 2018;14(28):1-12.

4. Lima ARA, Buss E, Ruiz MCS, González JS, Heck RM. Rural nursing formation possibilities: integrative review. Acta Paul Enferm. 2019;32(1):1139. http://dx.doi.org/10.1590/1982-0194201900016.

5. Pessoa VM, Almeida MM, Carneiro FF. Como garantir o direito à saúde para as populações do campo, da floresta e das águas no Brasil? Saúde Debate. 2018 set;42(spe1):302-14. http://dx.doi.org/10.1590/010311042018 s 120

6. Wünsch S, Denardin Budó MDL, Garcia RP, Oliveira SG, Gewehr M, Schimith MD et al. População rural e enfermagem: uma revisão bibliométrica. REUFSM. 2012;2(3):539-46. http://dx.doi.org/10.5902/217976923656.

7. Borges FA, Ogata MN, Feliciano AB, Fortuna CM. Management in health: the workers look of a rural family health. Cienc. Cuid. Saude. 2016;15(3):466-73. http://dx.doi.org/10.4025/cienccuidsaude.v15i3.31531.

8. Dimenstein M, Leite J, Macedo JP, Dantas C. Condições de vida e saúde mental em contextos rurais. Serv Soc Saúde. 2018;16(1):151-8. http://dx.doi.org/10.20396/sss.v16i1.8651478.

9. Coimbra Jr CEA. Saúde Rural Health in Brazil a still relevant old subject. Rev Saude Publica. 2018;52(1):1s-3s. http://dx.doi.org/10.11606/ S1518-8787.2018052000supl1ap.

10. Camargo BV, Bousfield ABS, Giacomozzi Al, Koelzer LP. Representações sociais e adesão ao tratamento antirretroviral. Liberabit [periódico na internet]. 2014; [citado 2020 nov 18];20(2):229-38. Disponível em: http:// www.scielo.org.pe/pdf/liber/v20n2/a04v20n2.pdf

11. Miranda No MV, Rewa T, Leonello VM, Oliveira MAC. Prática avançada em enfermagem: uma possibilidade para a Atenção Primária em 
Saúde? Rev Bras Enferm. 2018;71(Supl. 1):716-21. http://dx.doi. org/10.1590/0034-7167-2017-0672. PMid:29562033.

12. Hammerschmidt KSA, Mantovani MF, Labronici LM, Lenardt MH, Lacerda MR. The dissertations produced in the Graduate and Master's in Nursing Program at Universidade Federal do Paraná. Rev Esc Enferm USP. 2011;45(6):1466-75. http://dx.doi.org/10.1590/S008062342011000600027. PMid:22241208.

13. Lino MM, Backes VMS, Ferraz F, Reibnitz KS, Martini JG. Scientific production analysis of nursing education research groups in southern Brazil. Texto Contexto Enferm. 2010;19(2):265-73. http://dx.doi. org/10.1590/S0104-07072010000200007.

14. Schaurich D, Crossetti MGO. Knowledge production on nursing theories: analysis of the area periodicals, 1998-2007. Esc Anna Nery. 2010;14(1):182-8. http://dx.doi.org/10.1590/S1414-81452010000100027.

15. Barbosa Jr SA, Lira PVRA, Albuquerque PCC, Freitas AA. "O Jeca não é assim, ele está assim!": apontamentos sobre a história das políticas de saúde no meio rural do Brasil. Rev Ciênc Vet Saúde Públ. 2016;2(2):107-21. http://dx.doi.org/10.4025/revcivet.v2i2.28618.

16. Shimizu HE, Trindade JS, Mesquita MS, Ramos MC. Evaluation of the Responsiveness Index of the Family Health Strategy in rural areas. Rev Esc Enferm USP. 2018:52:e03316. http://dx.doi.org/10.1590/s1980220x2017020203316. PMid:29668788.

17. Ruiz ENF, Gerhardt TE. Políticas públicas no meio rural: visibilidade e participação social como perspectivas de cidadania solidária $e$ saúde. Physis. 2012;22(3):1191-209. http://dx.doi.org/10.1590/S0103 73312012000300018.

18. Ruiz ENF, Santos VF, Gerhardt TE. Mediações na atenção à saúde sob a ótica da Teoria da Dádiva: a saúde da população rural em destaque. Physis. 2016;26(3):829-52. http://dx.doi.org/10.1590/s010373312016000300007 .

19. Schwartz E, Lange $C$, Meincke SMK. A enfermagem e os cuidados à saúde da família rural. Fam. Saúde Desenv. 2001;3(1):48-53. http:// dx.doi.org/10.5380/fsd.v3i1.4946.

20. Oliveira AR, Sousa YG, Diniz ÍVA, Medeiros SM, Martiniano C, Alves M. The daily routine of nurses in rural areas in the Family Health Strategy. Rev Bras Enferm. 2019;72(4):918-25. http://dx.doi.org/10.1590/00347167-2018-0243. PMid:31432947.

21. Vieira F, Vieira R, Sá EC. Importância do enfermeiro do trabalho na promoção e prevenção de saúde frente às intoxicações por agrotóxicos em trabalhadores rurais. Saúde Ética Justiça. 2018;23(2):63-9. http:// dx.doi.org/10.11606/issn.2317-2770.v23i2p63-69.

22. Kessler Al, Krug SBF. From pleasure to suffering in the nursing work: the speech of the workers. Rev Gaúcha Enferm. 2012;33(1):49-55. http:// dx.doi.org/10.1590/S1983-14472012000100007. PMid:22737795.

23. Pitilin EB, Lentsck MH. Primary Health Care from the perception of women living in a rural area. Rev Esc Enferm USP. 2015;49(5):726-32. http:// dx.doi.org/10.1590/S0080-623420150000500003. PMid:26516740.

24. Budó MLD, Saupe R. Ways of care in rural communities: culture permeating the nursing care. Texto Contexto Enferm. 2005;14(2):177 85. http://dx.doi.org/10.1590/S0104-07072005000200004.

25. Arruda NM, Maia AG, Alves LC. Inequality in access to health services between urban and rural areas in Brazil: a disaggregation of factors from 1998 to 2008. Cad Saude Publica. 2018;34(6):e00213816. PMid:29947662.

26. Garnelo L, Lima JG, Rocha ESC, Herkrath FJ. Acesso e cobertura da Atenção Primária à Saúde para populações rurais e urbanas na região norte do Brasil. Saúde Debate. 2018;42(spe1):81-99. http://dx.doi. org/10.1590/0103-11042018s106.

27. Fernandes GCM, Boehs AE. A família rural em fases de transição: mudanças nos papéis e tarefas do cuidado familial. Cogitare Enferm. 2010;15(1):33-9. http://dx.doi.org/10.5380/ce.v15i1.17142.

28. Zillmer JGV, Schwartz E, Muniz RM. Nursing's view of the care practices of rural families and the person with cancer. Rev Esc Enferm USP. 2012;46(6):1371-8. http://dx.doi.org/10.1590/S0080-62342012000600013. PMid:23380780.

29. Wunsch S, Budó MLD, Girardon-Perlini NMO, Garcia RP, Oliveira SG. Care as a commitment of all: a study in a rural settlement. Rev Gaúcha Enferm. 2014;35(4):65-71. http://dx.doi.org/10.1590/19831447.2014.04.28526. PMid:25842782.
30. Ceolin T. Sistema de cuidado à saúde entre famílias rurais ao sul do Rio Grande do Sul [tese]. Pelotas: Universidade Federal de Pelotas; 2016.

31. Cavalcante IMS, Nogueira LMV. Health social collective for practices in the assentamento Mártires de Abril on island of the Mosqueiro Belém, Pará. Esc Anna Nery. 2008;12(3):492-9. http://dx.doi.org/10.1590/ S1414-81452008000300015.

32. Piriz MA, Mesquita MK, Cavada CT, Palma JS, Ceolin T, Heck RM Uso de plantas medicinais: impactos e perspectivas no cuidado de enfermagem em uma comunidade rural. Rev. Eletr. Enf. 2013;15(4):9929. http://dx.doi.org/10.5216/ree.v15i4.19773.

33. Peters CW, Lange C, Lemões MAM, Hermes GB, Linck CL, Carniére $\mathrm{CM}$. Conhecimentos, atitudes e práticas em saúde do idoso da zona rural. Rev Enferm UFPE on line [periódico na internet]. 2019; [citado 2020 nov 18];13(5):1465-74. Disponível em: https://periodicos.ufpe. $\mathrm{br} / \mathrm{revistas} / \mathrm{revistaenfermagem/article/view/238442}$

34. Morais EP, Rodrigues RAP, Gerhardt TE. Os idosos mais velhos no meio rural: realidade de vida e saúde de uma população do interior gaúcho. Texto Contexto Enferm. 2008;17(2):374-83. http://dx.doi.org/10.1590/ S0104-07072008000200021.

35. Winckler M, Boufleuer TR, Ferretti F, Sá CA. Idosos no meio rural: uma revisão integrativa. Estud Interdiscip Envelhec. 2016;21(2):173-94. http://dx.doi.org/10.22456/2316-2171.60691.

36. Tavares DMS, Fachinelli AMP, Dias FA, Bolina AF, Paiva MM. Preditores da qualidade de vida de idosos urbanos e rurais. Revista Baiana de Enfermagem. 2015;29(4):361-71. http://dx.doi.org/10.18471/rbe. v29i4.13359.

37. Santos EA, Tavares DMS, Rodrigues LR, Dias FA, Ferreira PCS. Morbidity and quality of life of elderly individuals with diabetes mellitus living in urban and rural areas. Rev Esc Enferm USP. 2013;47(2):393-400. http:// dx.doi.org/10.1590/S0080-62342013000200017. PMid:23743906.

38. Garbaccio JL, Tonaco LAB, Estêvão WG, Barcelos BJ. Aging and quality of life of elderly people in rural areas. Rev Bras Enferm. 2018;71(2, Supl. 2):724-32. http://dx.doi.org/10.1590/0034-7167-2017-0149. PMid:29791626.

39. Rodrigues LR, Tavares DMS, Silveira FCO, Dias FA, Martins NPF. Qualidade de vida, indicativo de depressão e número de morbidades de idosos da zona rural. Rev Enf Atenção Saúde [periódico na internet] 2015; [citado 2020 nov 18];4(2):33-44. Disponível em: http://seer.uftm. edu.br/revistaeletronica/index.php/enfer/article/view/1122/pdf

40. Buso ALZ, Viana DA, Alves LMS, Dias FA, Oliveira DV, Antunes $M D$ et al. Factors associated with life quality of the octogenarian elderly in the rural area. Cad Saude Colet. 2020;28(2):231-40. http://dx.doi. org/10.1590/1414-462x202000020193.

41. Corrêa ML, Carpena MX, Meucci RD, Silva LN. Depressão em idosos de uma região rural do Sul do Brasil. Cad Saude Colet. 2020;25(6):2083-92. http://dx.doi.org/10.1590/1413-81232020256.18392018. PMid:32520256.

42. Casagranda LP, Lange C, Zillmer JGV, Santos F, Castro DSP, Llano PMP. Prevalência de diabetes mellitus em idosos da zona rural no Sul do Brasil. Enfermagem Brasil. 2018;17(4):346-53. http://dx.doi. org/10.33233/eb.v17i4.990.

43. Llano PMP, Lange C, Nunes DP, Pastore CA, Pinto AH, Casagranda LP. Frailty in rural older adults: development of a care algorithm. Acta Paul Enferm. 2017;30(5):520-30.http://dx.doi.org/10.1590/1982-0194201700075.

44. FerreiraJP, Leeson G, MelhadoVR.Cartografias do envelhecimento em contexto rural: notas sobre raça/etnia, gênero, classe e escolaridade. Trab Educ Saúde. 2019;17(1):e0017612.http://dx.doi.org/10.1590/1981-7746-sol00176.

45. Pagliuca LMF, Lima BS, Silva JM, Cavalcante LM, Martins MC, Araújo TL. Acesso de idosos às unidades de Atenção Primária à Saúde. Rev Min Enferm. 2017;21:e-1021. http://dx.doi.org/10.5935/14152762.20170031.

46. Llano PMP, Lange C, Sequeira CAC, Jardim VMR, Castro DSP, Santos F. Factors associated with frailty syndrome in the rural elderly. Rev Bras Enferm. 2019;72(Supl. 2):14-21. http://dx.doi.org/10.1590/0034-71672017-0079. PMid:31826186 